\title{
Editorial
}

\section{Cannabinoids: for better and for worse}

\author{
Florence Thibaut, MD, PhD - Editor-in-Chief \\ Margret R. Hoehe, MD, PhD - Issue Coordinator
}

The use of cannabis as a drug has undergone a remarkable change of direction: considered as a symbol of countercultures in past decades, it is presently being hailed as a cure for any number of diseases and conditions. Thus, despite concerns about the safety of cannabis and cannabinoids, quite a few drugs that contain cannabinoids have recently been approved by several drug agencies, and the medicinal and recreational use of cannabis has been legalized in various countries and states. The promise of cannabinoids for therapeutic use, as well as potentially detrimental health risks and regulatory issues, will need to be carefully weighed.

C 2020, AICH - Servier Group

Dialogues Clin Neurosci. 2020;22(3):201-204. doi:10.31887/DCNS.2020.22.3/fthibaut

Keywords: cannabinoid; tetrahydrocannabinol (THC); cannabidiol; fatty acid amide hydrolase; endocannabinoid system; endocannabinoid signaling; cannabis use disorder (CBD); mental disease; psychosis; legalization; regulation; drug safety; health risk

Humans began to take advantage of the psychoactive properties of cannabis thousands of years ago. Today, more than 150 million people regularly smoke cannabis, making it one of the world's most popular recreational drugs ${ }^{1}$ (see also Crocq, in this issue, p 223). The major psychoactive ingredient of cannabis in the Cannabis sativa plant is delta-9tetrahydrocannabinol (THC), another important component is cannabidiol (CBD), and close to a hundred additional cannabinoids have been identified in the plant. As the primary target of THC, mediating its psychoactive effects, the cannabinoid type 1 receptor (CB1R) has been cloned, functionally expressed, ${ }^{2}$ and localized in the human genome, ${ }^{3}$ followed by the molecular characterization of the cannabinoid type 2 receptor (CB2R). ${ }^{4}$ Both $\mathrm{CB} 1 \mathrm{R}$ and $\mathrm{CB} 2 \mathrm{R}$, members of the $\mathrm{G}$ protein-coupled receptor (GPCR) gene family, represent the main receptors for the cannabinoids. Soon thereafter, endogenous ligands binding to these receptors were discovered, the "endocannabinoids," the best studied of which are anandamide or N-arachido- noylethanolamide (AEA) ${ }^{5}$ and 2-arachidonoylglycerol (2-AG). This rapid succession of key discoveries in the early 1990s spurred great interest in cannabinoid pharmacology and motivated the development of $\mathrm{CB} 1$ or $\mathrm{CB} 2$ receptor subtype-selective ligands, for example the $\mathrm{CB} 1$ receptor antagonist/inverse agonist SR141716A (rimonabant), launched for the management of obesity, and the neutral antagonist AM4113. As the first, highly potent and selective antagonist of the CB2 receptor, SR144528 was introduced. Finally, it should be mentioned that there exist (less known) non-CB1/non-CB2 receptors, with which THC, endocannabinoids, and several synthetic CB1R/CB2R agonists and antagonists can also interact.

In this context, cannabidiol (CBD) is of particular interest. While THC acts as a partial agonist of CB1R and may also bind to $\mathrm{CB} 2 \mathrm{R}, \mathrm{CBD}$ exerts more complex effects involving in addition other neurotransmitter and transduction signaling systems. Thus, besides its weak action on CB1R and CB2R,

Author affiliations: University Hospital Cochin, site Tarnier, Paris, France; Faculty of Medicine Paris Descartes (University of Paris); INSERM U1266, Institute of Psychiatry and Neuroscience, Paris, France, ORCID: 0000-0002-0204-5435 (Florence Thibaut); Department of Computational Molecular Biology, Max Planck Institute for Molecular Genetics, Berlin, Germany (Margret R. Hoehe). Address for correspondence: florence.thibaut@aphp.fr; hoehe@molgen.mpg.de 


\section{Editorial}

Cannabinoids: for better and for worse - Thibaut, Hoehe

it is also an agonist at the transient receptor potential cation channel subfamily V member 1 (TRPV1) and a negative allosteric modulator of CB1R. (As a net effect, $\mathrm{CBD}$, unlike THC, restrains rather than activates $\mathrm{CB} 1$ receptor signaling, and therefore does not induce the psychoactive effects of THC.) CBD also directly acts as positive allosteric modulator at various $\mathrm{GABA}_{\mathrm{A}}$ receptor subtypes. ${ }^{6}$ Moreover, $\mathrm{CBD}$ exerts action on receptors that mediate pain signaling and inflammation, and one of the main CBD targets is at least one receptor for the neurotransmitter serotonin, the $5 \mathrm{HT}_{1 \mathrm{~A}}$ receptor. ${ }^{7}$ Furthermore, CBD seems to bind to an orphan GPCR called GPR55, potentially mediating its antiepileptic effects. Importantly, CBD may inhibit the fatty acid amide hydrolase (FAAH), leading (indirectly) to an increase of endocannabinoids. In sum, CBD "wears many hats", 8 making it a promising candidate to develop therapeutic approaches for quite a number of diseases.

The receptors interacting with cannabinoids and endocannabinoids are widely distributed in the human brain. Specifically, CB1Rs are expressed in two main neuronal populations, the cortical glutamatergic and GABAergic forebrain neurons. In contrast, CB2Rs are mainly expressed in peripheral organs; certain CB2Rs, however, are also expressed postsynaptically in neuronal somatodendritic brain areas. The expression of CB2R can be increased under certain pathological conditions such as addiction, inflammation, anxiety, or epilepsy. Moreover, CB1R and CB2R knockout experiments, at least against certain genetic backgrounds, suggest that CBRs may play important roles in pathological conditions. Lutz, in this issue (p 207), elaborates the neurobiology of cannabinoid receptor signaling in great depth and breadth. Because, as he outlines, internal and external impacts on ECS components can both lead to dysregulation of ECS activity and subsequently to possible neuropsychiatric disorders, pharmacological intervention targeting the ECS appears to be a promising therapeutic approach. Spanagel, in this issue (p 241), comprehensively reviews the action of cannabinoids and the ECS in reward processing and the development of addictive behavior. Accordingly, understanding the underlying mechanisms allows drawing conclusions on potential pharmacological interventions, which target endocannabinoid signaling and are of promise for cannabis, alcohol, and opioid use disorders. (About $9 \%$ of the people who regularly use cannabis will develop a cannabis use disorder). ${ }^{9}$ Maldonado et al, in this issue (p 229), update recent advances on the function of the ECS in modulating fear, anxiety, and stress. Moreover, they review the pathophysiological role of each component of this system in the control of these protective emotional responses that also trigger pathological emotional disorders, and discuss future possible treatment strategies for these. Di Marzo, in this issue (p 259), expands the ECS to include several endocannabinoid-like lipid mediators, their metabolic enzymes, and their molecular targets to form the "endocannabinoidome." This complex signaling system is deeply involved in the development of major neuropsychiatric disorders and provides a substrate for future therapeutic drugs to treat these diseases, including, importantly, also noneuphoric plant cannabinoids, particularly cannabidiol. The endocannabinoidome also plays a role in the microbiota-gut-brain axis, which is emerging as an important actor in the control of affective and cognitive functions and in their pathological alterations. Complementarily, Fadda and coauthors, in this issue ( $\mathrm{p} 271$ ), will provide a summary of cannabinoid effects in support of possible therapeutic applications for major depression, bipolar disorder, anxiety, posttraumatic stress disorder, and schizophrenia, highlighting benefits and risks of cannabinoid use in the management of these illnesses. According to Morrison and Murray, in this issue ( $\mathrm{p} 251$ ), cannabis points to the synaptic pathology of mental disorders. Cannabis can elicit an acute psychotic reaction, and its long-term use is a risk factor for schizophrenia. The authors outline how aberrant synaptic components disrupt the highest psychological functions. They discuss the contrast between endocannabinoids which are important for learning and memory in the hippocampus and the impairment of hippocampal-dependent cognitive/ memory performance resulting from exogeneous CB1R agonist administration such as THC. Various other pro-psychotic compounds such as ketamine and methamphetamine share the same ability to disrupt synaptic signaling and the structural plasticity of dendritic spines in the hippocampus, basal ganglia or cerebral cortex.

The consumption of cannabis is particularly perilous where THC content varies greatly according to the type of preparation used. Thus, according to the EU Drug Market Report 2019 , THC content varies between $5 \%$ and $11 \%$ in herbal preparation/marijuana, between $7 \%$ and $18 \%$ in resin preparation/hashish, and up to $99 \%$ in crystalline synthetic THC or "spice" ${ }^{10}$ Similarly, the chemical analysis of "street" cannabis by the US Drug Enforcement Agency over the last two decades resulted in dramatically increased levels 


\section{Editorial}

Cannabinoids: for better and for worse - Thibaut, Hoehe

of THC which are 10 to 100 times greater than that of CBD. The increased THC content of cannabis may exacerbate the potential detrimental consequences of cannabis use, especially concerning cognitive and psychiatric side effects. ${ }^{11}$ Also, beginning cannabis use during early adolescence may lead to specific attentional dysfunction in adulthood. ${ }^{12}$ To emphasize other detrimental consequences of cannabis, smoking cannabis during pregnancy has been associated with lower birth weight, which is also reported for tobacco smoking during pregnancy. Cannabis may cause orthostatic hypotension and increase the risk of fainting and falls. It may increase the risk of road traffic accidents. Also, recurrent severe vomiting is reported in regular cannabis users. The use of pure synthetic THC may even lead to death. ${ }^{13}$

Moreover, the use of cannabinoids can produce adverse side effects when employed for therapeutic purposes. For instance, the use of CBD can be associated with diarrhea or somnolence, and may also result in abnormalities in liver function tests. The US Food and Drug Administration (FDA) has warned the public against using vaping products that contain THC. These products have been found to have been implicated in a large proportion of the reported cases of serious lung pathologies associated with vaping. When cannabis or its products are unintentionally consumed by children, this may lead to emergency-room admission. There have also been reports of contamination of cannabis or cannabinoid products with pesticides, microorganisms, or other substances. Finally, some cannabis-/cannabinoid-containing products may contain quantities of cannabinoids that are substantially different from what is stated on their labels, and therefore can be responsible for unexpected side effects.

Most studies on the endocannabinoid system (ECS) focus on anandamide and 2-AG. These are synthesized from membrane-associated lipid precursors, on-demand, act only locally, and are metabolized rapidly after intake, resulting in a short duration of action. Thus, compounds which affect the levels of endocannabinoids by increasing their halflife might be promising. 2-AG is a full agonist of CB1R and CB2R with moderate-to-low affinity. Anandamide is a high-affinity partial agonist of CB1R with low activity at CB2R. The basal level of 2-AG is approximately 1000 times higher than anandamide in the brain and may be the primary endogenous ligand for brain CBR.${ }^{14}$ In addition, 2-AG potentiates $\mathrm{GABA}_{\mathrm{A}}$ receptors, whereas anandamide is one of the vanilloid receptor agonists, also called the TRPV1 agonist. ${ }^{15}$ These endocannabinoids retrogradely stimulate $\mathrm{CB} 1$ receptors on presynaptic terminals, leading to short- or long-term suppression of neurotransmitter release. Their overall effect is either excitatory or inhibitory depending on the presynaptic inhibition of GABAergic or glutamatergic transmission, respectively. Then, GABA inhibition may result in disinhibition of dopamine transmission in the ventral tegmental area; endocannabinoids may also interact with glutamate in the nucleus accumbens; both interactions may contribute to reward (for review see Spanagel in this issue, p 241). Fatty acid amide hydrolase (FAAH) is the principal catabolic enzyme of anandamide. Specific inhibitors of this enzyme have been studied in order to increase the level of endocannabinoids and as potential medications for various disorders such as pain, inflammation, and anxiety, as well as cancer. ${ }^{16}$ However, according to Habib et al, ${ }^{17}$ the inhibition of the expression of the newly identified pseudogene FAAH-OUT constitutes a much more promising therapeutic avenue to induce an analgesic and anxiolytic effect than that of the inhibitory molecules of the enzyme FAAH which have failed in humans and even led to severe side effects.

Despite the concerns about the safety of cannabis and cannabinoids as mentioned above, several drugs that contain cannabinoids were recently approved by drug agencies. Thus, cannabidiol was approved by both the FDA and the European Medicines Agency (Di Marzo, in this issue, p 259) for the treatment of seizures associated with Lennox-Gastaut syndrome or Dravet syndrome, two rare and severe forms of epilepsy. Dronabinol and nabilone (synthetic THC) were approved by the FDA to treat nausea and vomiting associated with cancer chemotherapy. Dronabinol is also used in case of loss of appetite and weight related to AIDS. Modest benefits of cannabinoids were also reported in patients with chronic pain or multiple sclerosis. Due to the adverse effects inevitably linked to the stimulation of $\mathrm{CB} 1$ receptors, researchers have preferentially looked for compounds that may target $\mathrm{CB} 2$ or non $\mathrm{CB} 1 /$ non $\mathrm{CB} 2$ receptors, or also compounds that may increase the level of endocannabinoids which may act as neuromodulators. At present, numerous clinical trials that are investigating potential medical benefits of many chemical components of cannabis are painting a promising picture. ${ }^{18}$

Hall, in this issue ( $\mathrm{p} 281$ ) will discuss the effects of cannabis legalization in Canada and the US, as well as their poten- 


\section{Editorial}

Cannabinoids: for better and for worse - Thibaut, Hoehe

tial consequences for public health. The risk of increased cannabis use and cannabis use disorders and their potential health consequences for the general population, is likely to increase, at least in the most fragile and precarious populations. This was already observed worldwide with alcohol and tobacco. The very powerful alcohol and tobacco industry and the revenue generated by taxes for the governments make it difficult to reverse legalization once the decision is made. Marcu (p 289) will round off this issue, covering recent advances in cannabis and cannabinoid regulation and drug approval. He advocates multiple pathways for product approval that adapt to the diversity of the products and the needs of the consumer. Hopefully these pathways would protect public health, whether the public is using cannabis and cannabinoids as a medicine, a wellness product, or as a recreational substance.

\section{References}

1. Lawler A. Oldest evidence of marijuana use discovered in 2500-year-old cemetery in peaks of western China. Science. 2019. Available at: https://www. sciencemag.org/news/2019/06/oldest-evidence-marijuana-use-discovered-2500-year-old-cemetery-peaks-western-china. Accessed August 2020.

2. Matsuda LA, Lolait SJ, Brownstein MJ, Young AC, Bonner TI. Structure of a cannabinoid receptor and functional expression of the cloned cDNA. Nature. 1990;346:561-564. doi:10.1038/346561a0.

3. Hoehe MR, Caenazzo L, Martinez MM, et al. Genetic and physical mapping of the human cannabinoid receptor gene to chromosome 6q14-q15. New Biol. 1991;3(9):880-885.

4. Munro S, Thomas KL, Abu-Shaar M. Molecular characterization of a peripheral receptor for cannabinoids. Nature. 1993;365(6441):61-65. doi:10.1038/365061a0.

5. Devane WA, Hanus L, Breuer A, et al. Isolation and structure of a brain constituent that binds to the cannabinoid receptor. Science. 1992;258(5090):1946-1949. doi:10.1126/science.1470919.

6. Bakas T, van Nieuwenhuijzen PS, Devenish SO, McGregor IS, Arnold JC, Chebib M. The direct actions of cannabidiol and 2-arachidonoyl glycerol at GABA receptors. Pharmacol Res. 2017;119:358-370.

7. Fogaça MV, Reis FM, Campos AC, Guimarães FS. Effects of intra-prelimbic prefrontal cortex injection of cannabidiol on anxiety-like behavior: involvement of 5HT1A receptors and previous stressful experience. Eur Neuropsychopharmacol. 2014;24:410-419.

8. Eisenstein M. The reality behind cannabidiol's medical hype. Nature Outlook. 2019. Available at: https://www.nature.com/articles/d41586-019-02524-5. Accessed August 2020.

9. Lopez-Quintero C, Pérez de los Cobos J, Hasin DS, et al. Probability and predictors of transition from first use to dependence on nicotine, alcohol, cannabis, and cocaine: results of the National Epidemiologic Survey on Alcohol and Related Conditions (NESARC). Drug Alcohol Depend. 2011;115(1-2):120-130.

10. EU drug markets report 2019. Updated on $9^{\text {th }}$ January 2020. Available at: https://www.emcdda.europa.eu/publications/joint-publications/eu-drug-marketsreport-2019 en. Accessed August 2020.

11. ElSohly MA, Mehmedic Z, Foster S, Gon C, Chandra S, Church JC. Changes in cannabis potency over the last two decades (1995-2014) - analysis of current data in the United States. Biol Psychiatry. 2016;79(7):613-619.

12. Ehrenreich H, Rinn T, Kunert HJ, et al. Specific attentional dysfunction in adults following early start of cannabis use. Psychopharmacology (Berl). 1999;142(3):295-301. doi:10.1007/s002130050892.

13. European Monitoring Centre for Drugs and Drug Addiction. 2017. Synthetic cannabinoids in Europe (perspectives on drugs). Available at: https://www. emcdda.europa.eu/publications/pods/synthetic-cannabinoids. Published June 2017. Accessed July 2020.

14. Zou S, Kumar U. Cannabinoid receptors and the endocannabinoid system: signaling and function in the central nervous system. Int J Mol Sci. 2018;19(3): 833-856. doi:10.3390/ijms19030833.

15. Di Marzo V, De Petrocellis L. Endocannabinoids as regulators of transient receptor potential (TRP) channels: a further opportunity to develop new endocannabinoid-based therapeutic drugs. Curr Med Chem. 2010;17:1430-1449.

16. Feledziak M, Lambert DM, Marchand-Brynaert J, Muccioli GG. Inhibitors of the endocannabinoid-degrading enzymes, or how to increase endocannabinoid's activity by preventing their hydrolysis. Rec Pat CNS Drug Discov. 2012;7:49-70.

17. Habib AM, Okorokov AL, Hill MN, et al. Microdeletion in a FAAH pseudogene identified in a patient with high anandamide concentrations and pain insensitivity. Br J Anaesth. 2019;123(2):e249-e253.

18. Drew L. Cannabis research round-up. Highlights from laboratory studies and clinical trials. Nature Outlook. 28 August 2019. Available at: https://www. nature.com/articles/d41586-019-02532-5. Accessed August 2020. 